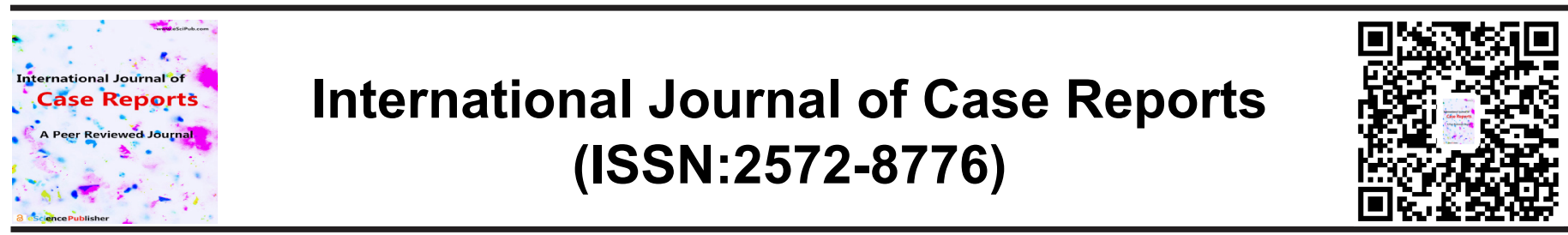

\title{
Giant gastric carcinosarcoma infiltrating the left hepatic lobe: a case report
}

\section{Leonardo Vincenti MD ${ }^{1}$, Cinzia Bizzoca MD¹, Salvatore Fedele MD $^{2}$, Anna Maria Valentini $\mathrm{BScD}^{3}$, Valeria Andriola ${ }^{1}$, Graziana Arborea $\mathrm{MD}^{3}$}

${ }^{1}$ General Surgery "Ospedaliera”, Policlynics of Bari, Italy; ${ }^{2}$ Dept of General Surgery, National Institute of Gastroenterology "S. de Bellis", Research Hospital, CastellanaGrotte (BA), Italy; ${ }^{3}$ Dept of Pathology, National Institute of Gastroenterology "S. de Bellis", Research Hospital, Castellana Grotte (BA), Italy

\section{ABSTRACT}

Gastric carcinosarcoma is a rare biphasic tumor that consists of both carcinomatous and sarcomatous components. Clinical presentation and neoplastic markers are not specific for the disease. Moreover, CT scan cannot distinguish among gastric carcinoma, sarcoma or carcinosarcoma, so it is essential to perform histopathological and immunohistochemical analysis on biopsy specimens or resected tissue to ensure a correct diagnosis.

A 72-year-old woman was transferred to our Department from another hospital with a diagnosis of a bleeding tumor localized on the lesser gastric curve, infiltrating the left hepatic lobe.

The mass was treated as a gastric malignant mesenchymal neoplasia (gastro-intestinal stromal tumor versus sarcoma) infiltrating the liver. Owing to the bleeding, no further invasive examinations, such as fine needle aspiration biopsy, were planned. Furthermore, no neoadjuvant chemotherapy could be administered before surgery due to persistent anaemia.

The scheduled surgical procedure was an atypical gastric resection with "en bloc" hepatic resection. This operation included upper polar and lesser curve resection, with tubulization of the stomach along the greater curve, as well as left lateral hepatic sectionectomy.

Histopathological examination and immunohistochemical staining confirmed the diagnosis of gastric carcinosarcoma.

This is first report of gastric carcinosarcoma with local liver infil*Correspondence to Author: Cinzia Bizzoca M.D.

Department of General Surgery "Ospedaliera". Polyclinic Hospital of Bari, Bari, Italy. Piazza G. Cesare, 11 - 70124 - Bari (BA). Italy.

How to cite this article:

Leonardo Vincenti, Cinzia Bizzoca, Salvatore Fedele, Anna Maria Valentini, Valeria Andriola, Graziana Arborea. Giant gastric carcinosarcoma infiltrating the left hepatic lobe: a case report. International Journal of Case Reports, 2021 $5: 214$ tration, treated with surgical resection.

Keywords: Gastric Carcinosarcoma, Gastrectomy, Hepatic resection

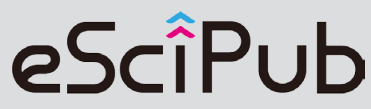

eSciPub LLC, Houston, TX USA. Website: http://escipub.com/ 


\section{Introduction}

Gastric cancer is the most common malignancy and the third cause of cancer-related death. Most malignant gastric tumors (more than 90\%) are classified as adenocarcinoma, whereas carcinosarcoma (CS) of the stomach is a rare, aggressive disease ${ }^{1}$. It is a rare biphasic tumor which consists of both carcinomatous and sarcomatous components ${ }^{2}$. Carcinosarcomas usually arise in other organs, such as the uterus, breast, thyroid and lung ${ }^{3-5}$. When they occur in the gastrointestinal tract, the esophagus is the most common site of origin 5 . Instead, the stomach is a rare localization of this uncommon neoplasm, as well as the liver ${ }^{6-9}$. Histologically, carcinosarcoma can be subdivided into three types, according to its origin. Type 1 is the "collision" tumor, where there is a clear boundary between the epithelial and stromal components. Type 2 is the "combined" tumor, where neoplastic cells show overlapping of epithelial and stromal characteristics, although the stromal component does not have any distinguishing characteristics. In type 3 or "composite" tumors, both neoplastic types are detected, and the stromal components show different characteristics ${ }^{4}$.

In 1904, Quecken-stadt first reported a case of gastric carcinosarcoma ${ }^{4}$. An Italian group described five cases of carcinosarcoma of the stomach during a 10 -year period ${ }^{10}$. Overall, less than 100 cases of primary gastric CS have been reported in literature since Queckenstadt's report in 190411, and none of the authors described local infiltration of the liver. The reported cases were prevalently males, with a median age of 62 years ${ }^{5}$. Clinical presentation and neoplastic markers are not specific for the disease, so carcinosarcoma and gastric adenocarcinoma forms do not differ, and an endoscopic or radiological discriminating diagnosis is impossible ${ }^{5}$. In fact, CT scan cannot distinguish among gastric carcinoma, sarcoma or CS. To ensure a correct diagnosis, it is essential to perform histopathological and immunohistochemical analysis on biopsy specimens or resected tissue ${ }^{5,8}$.

The AJCC/UICC TNM staging system does not provide a specific classification for gastric CS but it is utilized by some authors based on the disease stage of the carcinoma component. Unfortunately, patients are often diagnosed at an advanced stage, when the prognosis is worse ${ }^{12}$.

\section{Case report}

A 72-year-old woman was transferred to our Department from another hospital with a diagnosis of a tumor localized on the lesser gastric curve, infiltrating the left hepatic lobe.

The clinical presentation was unusual, because the patient was studied for fever of unknown origin, lasting about one month and associated with non-specific weight loss. The patient had undergone several tests, none of which were pathognomonic for gastric tumor. CEA and CA 19.9 levels were normal, whereas CA 15.3 and CA 125 were twice the normal values. Nonspecific persistent anaemia was treated with repeated blood transfusions. Additionally, several antibiotics were administered, but failed to resolve the fever. When the patient presented melaena, abdominal pain and dysphagia, abdominal CT scan and EGDS with biopsy were performed. The CT scan detected a complex mass measuring $11 \times 8 \mathrm{~cm}$, originating from the lesser gastric curve and infiltrating the II and III hepatic segments (Figure 1). The radiologist diagnosed a suspected malignant gastrointestinal stromal tumor (GIST) or leiomyosarcoma. This diagnosis was supported by esophago-gastric endoscopy, which revealed a large gastric mass with mucosal ulceration, without infiltration of the gastric mucosa at biopsy.

At the time of admission to our ward, the patient presented fever, anaemia and a poor nutritional and general status. Parenteral nutrition, fluid therapy and blood transfusion were administered, in order to correct altered serum values. The fever was controlled by corticosteroid administration. Because of the 
bleeding, no further invasive examinations, such as fine needle aspiration biopsy (FNAB), were planned. In any case, biopsy was not mandatory, since no neoadjuvant chemotherapy could be administered before surgery, due to the persistent anaemia and poor performance status. After multidisciplinary discussion, the mass was treated as a gastric malignant mesenchymal tumor locally infiltrating the liver, in the absence of distant metastases, therefore we opted for upfront surgery.

At laparotomy, the huge gastric mass entirely occupied the lesser curve up to the right subcardial region. Furthermore, as expected, the II e III hepatic segments were infiltrated. No ascites or distant metastases were detected at exploration. The operation consisted of upper polar and lesser curve resection, with tubulization of the stomach along the greater curve. The mass was resected "en bloc" together with left lateral hepatic sectionectomy. The gastrointestinal continuity was restored through a mechanical termino-lateral esophago-gastric tube anastomosis.

The postoperative course was uneventful in the first days, but we preferred to perform a radiological examination of the anastomosis before starting oral intake.

We used Gastrographin as contrast medium, to gain a full picture of the functional outcome, in addition to documenting the absence of fistulas (Figure 2). The patient was gradually started on an oral diet and was discharged on POD (postoperative day) 9 , with instructions to continue a semi-liquid diet for one month. Unfortunately she survived only 5 months, due to neoplastic recurrence.
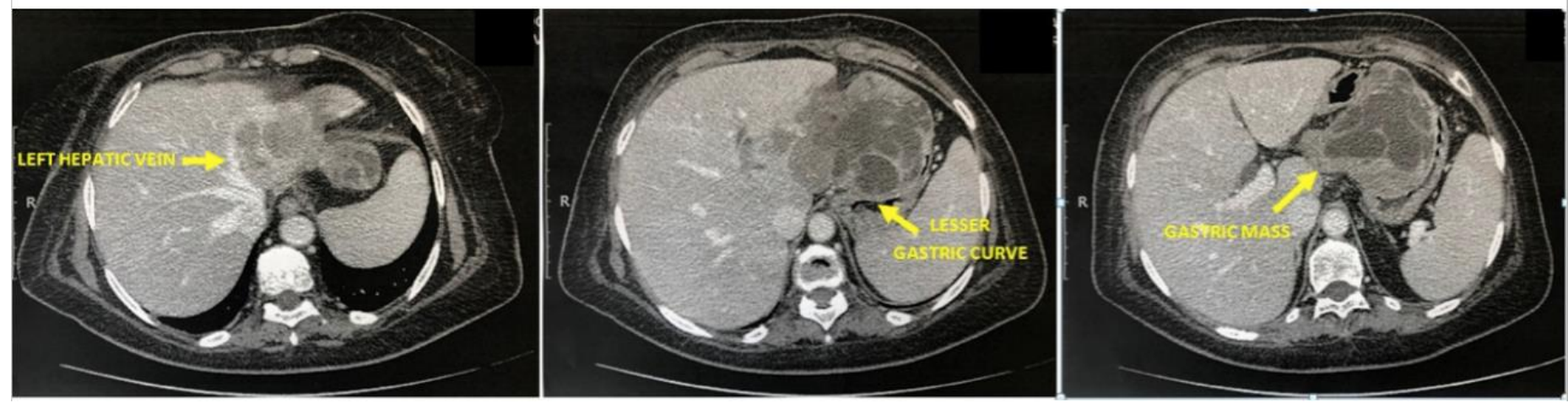

Fig.1. Abdomen CT scan with contrast shows gastric mass compression on left hepatic vein and stomach (arrows).
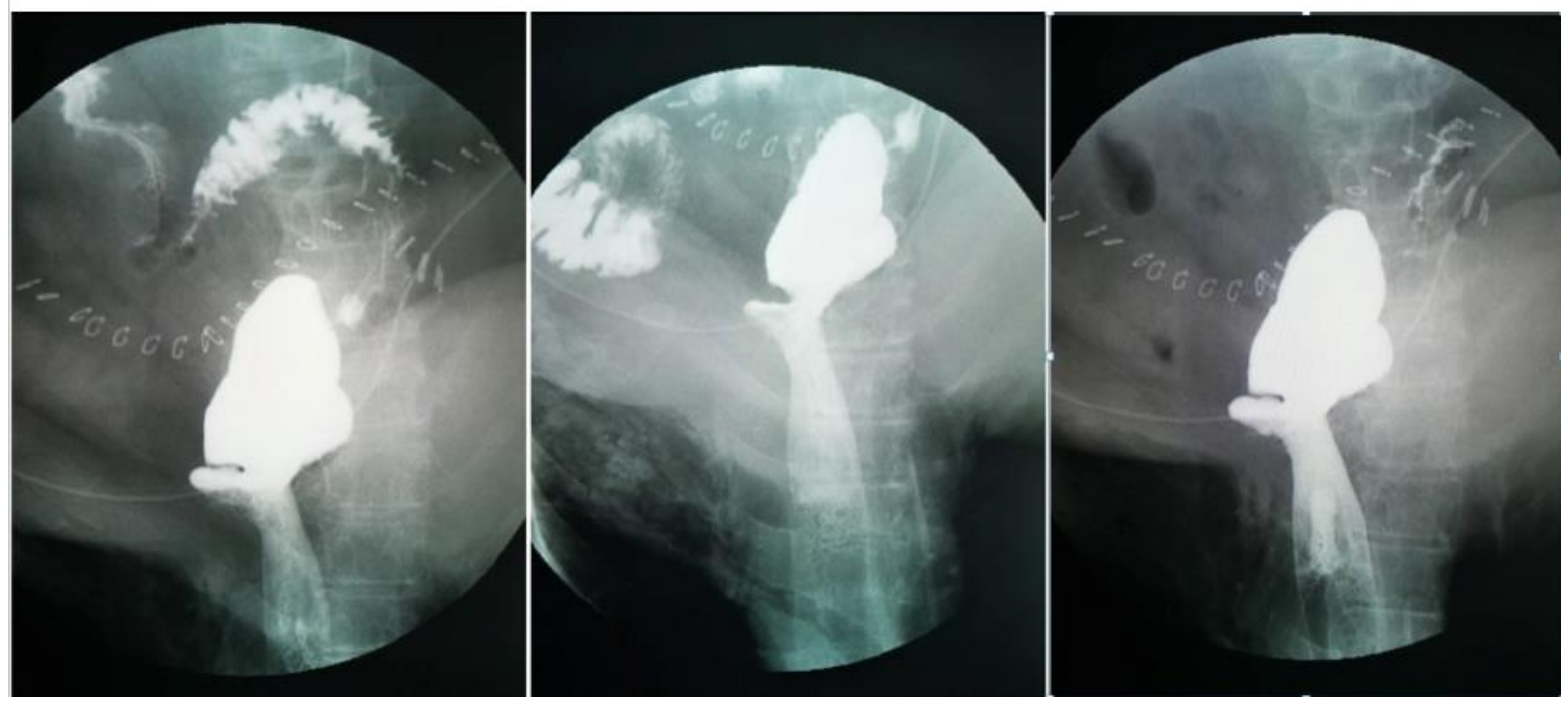

Fig.2. Contrast esophagogastric radiological examination: neither fistula nor stenosis were detected 


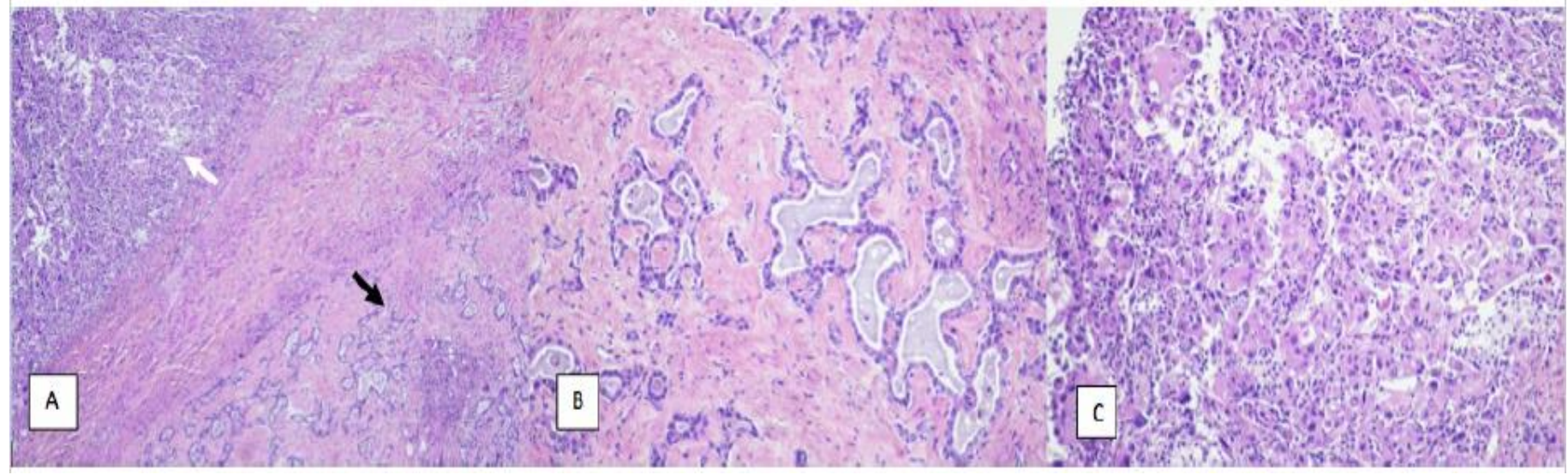

Fig.3. Photomicrographs of hematoxylin-eosin-stained tissue sections of the tumor. (A) The tumor consisted of both carcinoma (black arrow) and sarcoma ( white arrow) components . (B-C) Higher magnification showing neoplastic glands (B) and sarcomatous component (C).

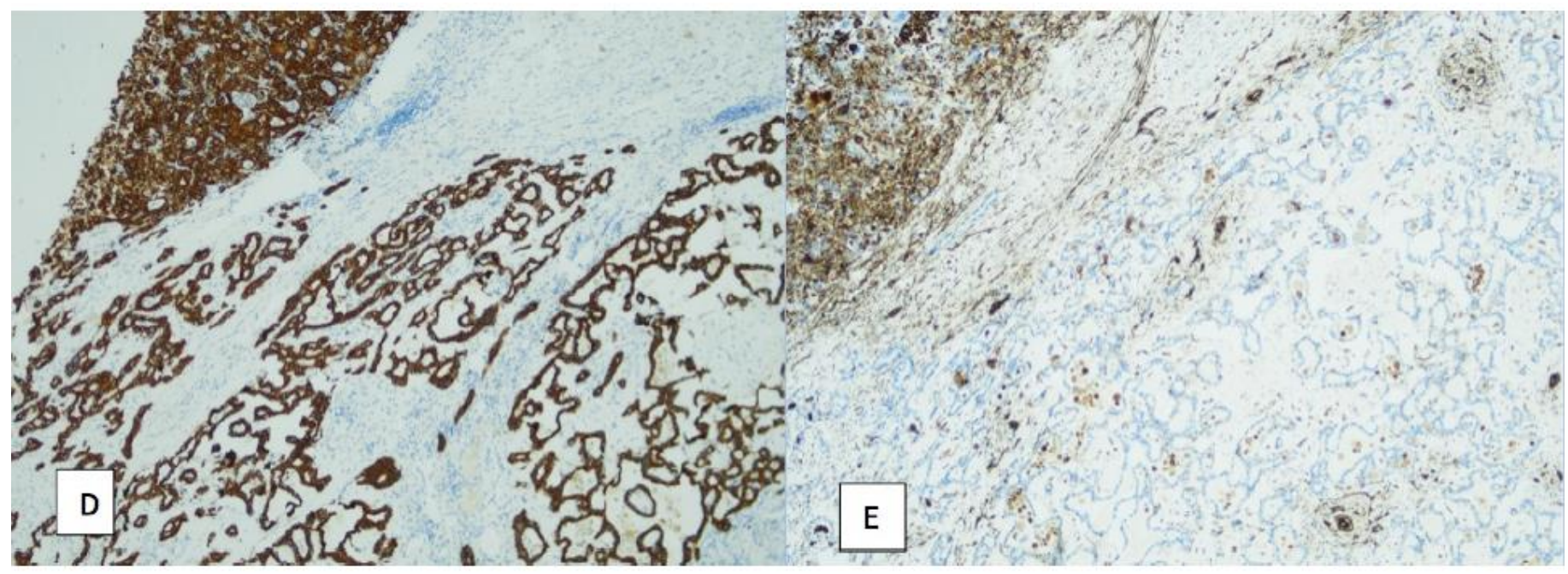

Fig.4. (D) Immunohistochemical staining showing the expression of pan cytokeratins in both adenocarcinomatous and sarcomatous components and (E) of Vimentin only in sarcomatous one.

\section{Pathology}

Macroscopically, the tumor sample measured 14 $\mathrm{cm}$ in maximum diameter, was partially covered by $10 \mathrm{~cm}$ of gastric mucosa and extended $10 \mathrm{~cm}$ into the left hepatic lobe. The esophageal and gastric rings obtained after mechanical anastomosis were also analyzed.

The cut face showed a solid lesion with a whitish-brown color, soft consistency and necrotic central area.

Histopathologically, a malignant, poorly differentiated tumor involving the gastric wall was detected. Microscopic examination by conventional hematoxylin-eosin staining showed that the tumor included both a carcinomatous and sarcomatous component, that were clearly distinct (Figure 3).

The carcinomatous component was composed of a moderately differentiated adenocarcinoma, whereas the sarcomatous component mainly consisted of polygonal cells with prominent nucleoli, and large, hyperchromatic nuclei with an eosinophilic cytoplasm. Several nuclear pleomorphisms and extensive necrotic areas were seen. Numerous mitotic figures, often atypical, were also detected. There were no lymph node metastases nor venous invasion. The tumor extended to the left hepatic lobe, but on the other side it did not reach the gastric 
mucosa. Surgical resection margins were not infiltrated by the tumor.

Immunohistochemical (IHC) staining was carried out to confirm the diagnosis, showing IHC immunoreactivity to pan cytokeratins AE1/AE3 in both the adenocarcinomatous and sarcomatous components. Furthermore, the carcinomatous cells showed a strong positivity to cytokeratin 7 whereas the sarcomatous cells showed diffuse staining for vimentin (Figure 4).

IHC staining for glypican 3 was performed in order to exclude hepatic histogenesis of the neoplasm, while IHC staining for DOG1, cKit, CD34 and Myogenin was done to exclude a GIST diagnosis.

Morphological and IHC findings confirmed the diagnosis of gastric carcinosarcoma. Additionally, Smooth Muscle Actin, HHF35, Caldesmon, Desmin, CD68, Chromogranin A and Synaptophysin were performed to better characterize both tumor components but all markers resulted negative.

\section{Discussion}

Herein, we report the first case of gastric carcinosarcoma with local liver infiltration, treated with surgical resection.

CS is an aggressive tumor, with a worse prognosis than gastric carcinoma even if radical treatment is possible. The mean survival period is estimated to be $10-15$ months, while more than $50 \%$ of tumor recurrences occur within the first postoperative year, mainly in the peritoneum and liver ${ }^{4,5,13}$.

Clinical presentation is not specific for the disease, as the symptoms are similar to gastric carcinoma, featuring loss of appetite, abdominal pain, dysphagia or anaemia in cases of mucosal bleeding ${ }^{3}$. Neoplastic markers levels (CEA, CA 19.9) are also non-specific. CT scan may reveal a small or huge lesion of the gastric wall, with or without lymph nodes or liver metastases. The correct diagnosis is often reached only after surgery, after histopathological and immunohistochemical analysis of the resected tumor ${ }^{5}$.
In our case, both conventional hematoxylineosin staining (Figure 3 ) and IHC findings (Figure 4) seemed to confirm a type $1 \mathrm{CS}$.

Two main hypotheses have been formulated to explain the histogenesis of CS. The first is the stem cell theory, whereby CS is derived from a single totipotent stem cell, which is able to differentiate toward both epithelial and mesenchymal metaplasia and neoplasia ${ }^{14}$.The second is the tumor collision theory, whereby CS originates from a collision between epithelial and mesenchymal neoplastic cells ${ }^{15}$. According to some authors, the primary carcinoma may stimulate excessive stromal proliferation, resulting in a carcinosarcoma. According to another theory, the spindle cell component of CS is not of mesenchymal origin, but reflects anaplasia within the carcinomatous neoplasia 16,17. Finally, the theories could all be true, as all gastric biphasic tumors are of epithelial origin, but with the potential to give rise to different types of $\mathrm{CS}^{18}$.

Radical treatment of CS is total gastrectomy with D2 lymphectomy. Nonetheless, palliative gastrectomy or subtotal gastrectomy are also considered adequate options, in order to control gastric symptoms such as bleeding or stenosis ${ }^{5}$.

In the case we report, the patient suffered from anaemia due to chronic bleeding and a lateonset mass effect, with consequent abdominal pain and dysphagia. After multidisciplinary debate, we decided to avoid preoperative FNAB, in order to prevent worsening the tumoral bleeding. Furthermore, since EGDS and mucosal biopsy were negative for gastric carcinoma, a mesenchymal tumour was suspected. This diagnosis was further confirmed by radiological findings, since the radiologist suggested a diagnosis of malignant GIST or sarcoma of gastric origin. The definitive diagnosis of CS is often obtained only on the resected tissue, because the limited specimen retrieved by FNAB is frequently not adequate to diagnose this rare neoplasm ${ }^{5}$.

The scheduled surgical procedure consisted of atypical gastrectomy "en bloc" with left lateral 
hepatic sectionectomy. The surgery goal was tumor resection with negative margins, in order to resolve the symptoms and allow adjuvant therapies. Due to the rare incidence of the disease, there is no standard chemotherapy regimen, so no adjuvant therapy was administered.

\section{Conclusion}

Gastric carcinosarcoma is a rare, aggressive tumor, that is difficult to diagnose correctly before surgical resection. It is sometimes detected because of complications, such as bleeding. The early symptoms are non-specific, whereas the definitive diagnosis is often only possible after IHC staining of the resected tissue. Due to the rarity of the disease, nonspecific symptoms as well as poor prognosis, both the diagnosis and treatment of CS are very challenging.

Abbreviations: CT computed tomography, CS Carcinosarcoma, EGDS Esophago-GastroDuodenoscopy, GIST Gastro-Intestinal Stromal Tumour, FNAB fine needle aspiration biopsy, POD Post-operative day

\section{References}

[1]. Torre LA, Bray F, Siegel RL, Ferlay J, LortetTieulent J, Jemal A. Global cancer statistics, 2012. CA: A Cancer Journal for Clinicians. 2015;65(2). doi:10.3322/caac.21262

[2]. CIROCCHI R, TRASTULLI S, DESIDERIO J, et al. Gastric carcinosarcoma: A case report and review of the literature. Oncology Letters. 2012;4(1). doi:10.3892/ol.2012.699

[3]. Bekki T, Fujikuni N, Tanabe K, et al. The gastric carcinosarcoma with severe venous invasion: a case report. Surgical Case Reports. 2018;4(1). doi:10.1186/s40792-018-0421-8

[4]. Choi KW, Lee WY, Hong SW, Chang YG, Lee B, Lee HK. Carcinosarcoma of the Stomach: A Case Report. Journal of Gastric Cancer. 2013;13(1). doi:10.5230/jgc.2013.13.1.69

[5]. Lazaridis II, Lazaridis L-D, Spartalis E, Frountzas M, Schizas D. Gastric carcinosarcoma: a rare clinical entity looking for an identity. JBUON. 2018;23(5):1262-1265.

[6]. Fujiie M, Yamamoto M, Taguchi K, et al. Gastric carcinosarcoma with rhabdomyosarcomatous differentiation: a case report and review. Surgical Case Reports. 2016;2(1). doi:10.1186/s40792016-0176-z
[7]. Selcukbiricik F, Tural D, Senel ET, Dervisoglu S, Serdengecti S. Gastric carcinoma with osteoblastic differentiation. International Journal of Surgery Case Reports. 2012;3(11). doi:10.1016/j.jijscr.2012.07.001

[8]. Kuroda H, Saito H, Kono Y, et al. Carcinosarcoma of stomach confined to the mucosa. Yonago Acta Medica. doi:10.24563/yam.2017.12.006 2017;60(4):246-250.

[9]. Li J, Liang P, Zhang D, et al. Primary carcinosarcoma of the liver: imaging features and clinical findings in six cases and a review of the literature. Cancer Imaging. 2018;18(1). doi:10.1186/s40644-018-0141-0

[10]. Wronski M. Synchronous occurrence of gastrointestinal stromal tumors and other primary gastrointestinal neoplasms. World Journal of Gastroenterology. doi:10.3748/wjg.v12.i33.5360

[11]. Nie L, Zhou X, Peng L, Fu Y, Cheng Y, Huang Q. Histological heterogeneity and distributional difference of gastric carcinosarcoma: Report of 4 cases and literature review. Polish Journal of Pathology. 2018;69(4):366-375. doi:10.5114/pjp.2018.81696

[12]. Gohongi T. Postsurgical radiation therapy for gastric carcinosarcoma with c-kit expression: A case report. World Journal of Gastroenterology. 2015;21(9). doi:10.3748/wjg.v21.i9.2830

[13]. Ochiai A, Teramachi K, Kanomata N, Hasebe T, Ishii G, Sugito M. Carcinosarcoma (pure endocrine cell carcinoma with sarcoma components) of the stomach. Pathology International. 2003;53:552-556. doi:10.1046/j.1320-5463.2003.01508.x

[14]. Liu S-W, Chen G-H, Hsieh P-P. Collision Tumor of the Stomach. Journal of Clinical Gastroenterology. 2002;35(4). doi:10.1097/00004836-200210000-00010

[15]. Randjelovic T. Carcinosarcoma of the stomach: A case report and review of the literature. World Journal of Gastroenterology. 2007;13(41). doi:10.3748/wjg.v13.i41.5533

[16]. Carcangiu ML, Steeper T, Zampi G, Rosai J. Anaplastic Thyroid Carcinoma: A Study of 70 Cases. American Journal of Clinical Pathology. 1985;83(2). doi:10.1093/ajcp/83.2.135

[17]. Tokunaga $O$, Morimatsu M, Nakashima T. COLLISION TUMOR OF THE STOMACH WITH CARCINO-SARCOMA AND TUBULOPAPILLARY ADENOCARCINOMA. Pathology International. 1979;29(5). doi:10.1111/j.14401827.1979.tb00947.x

[18]. Kayaselçuk F, Tuncer İ, Toyganözü Y, Bal N, Özgür G. Carcinosarcoma of the stomach. Pathology\&OncologyResearch. 2002;8(4). doi:10.1007/BF03036745 